\title{
Ansia e paura della recidiva in pazienti affetti da tumore differenziato della tiroide nonostante la buona prognosi
}

\author{
Emanuela Arvat ${ }^{1}$
}

Pubblicato online: 29 gennaio 2018

(c) Springer International Publishing AG, part of Springer Nature 2018

Commento a:

Anxiety and fear of recurrence despite a good prognosis: an interview study with differentiated thyroid cancer patients.

C. Hedman, P. Strang, T. Djarv, I. Wildberg, C.I. Lundgren.

Thyroid (2017) 27:1417-1423

L'impatto del cancro sulla sfera emotiva dell'individuo è ormai riconosciuto e accertato dalla letteratura scientifica. Ansia e/o depressione sono le conseguenze più comuni in cui può incorrere la popolazione dei malati di cancro. Le ricerche degli ultimi anni hanno manifestato un crescente interesse nella valutazione dell'impatto della malattia cancro e dei suoi trattamenti sulla qualità di vita del paziente. Per quanto concerne il tumore differenziato della tiroide (DTC), è stato evidenziato come pazienti con pregressa diagnosi di questo tipo di tumore abbiano, nonostante una prognosi generalmente favorevole, problematiche emotive molto simili ai sopravvissuti di altri tipi di neoplasie.

Lo scopo di questo studio è stato quello di valutare la presenza e il livello di ansia, le possibili motivazioni e le strategie di controllo della stessa in un gruppo di pazienti con DTC.

Ventun pazienti ( 8 maschi e 13 femmine, età media 49 anni) sono stati inclusi nello studio. Sono state somministrate interviste semi-strutturate, registrate su nastro e trascritte testualmente, analizzate con un'analisi del contenuto qualitativa. I dati sono stati valutati in relazione a età, sesso,

\section{E. Arvat}

emanuela.arvat@unito.it

1 Divisione di Endocrinologia e Malattie del Metabolismo, Dipartimento di Medicina Interna, Ospedale Molinette, Università di Torino, Torino, Italia stadio della malattia, livello d'istruzione e tempo trascorso dalla diagnosi.

Dai dati raccolti è emerso che sia i pazienti con recidive sia quelli senza recidive manifestavano uno stato di ansia correlata alla loro condizione clinica al momento dell'intervista. La preoccupazione per il futuro, la paura della recidiva e di sviluppare altri tumori, il doversi sottoporre a continui accertamenti e la possibile mancanza di ulteriori opzioni di trattamento rappresentavano dei punti importanti nel generare lo stato emotivo ansioso. Tali dati sono risultati indipendenti da parametri socio-demografici, tempo intercorso dalla diagnosi e diversità dei trattamenti subiti in precedenza. È stato tuttavia interessante rilevare che inizialmente sono stati minimizzati o addirittura negati lo stato di ansia e le motivazioni correlate, espressi invece alla fine delle interviste. Per far fronte all'ansia, i pazienti hanno sviluppato strategie protettive come concentrarsi sulla routine quotidiana, sulle distrazioni e concentrarsi sulle "piccole cose nella vita".

I dati dello studio, sebbene eseguito su un numero esiguo di pazienti, evidenziano chiaramente come la condizione emotiva di ansia sia comune nei pazienti affetti da DTC, indipendentemente dal livello di gravità, dalle tipologie dei trattamenti e dal tempo intercorso dalla diagnosi. In particolare, l'atteggiamento di negazione del sintomo all'inizio delle interviste indica la necessità di un tempo adeguato di colloquio con tali pazienti; inoltre, l'evidenza che l'ansia sia associata al follow-up induce alla rivalutazione dei tempi e delle modalità di monitoraggio della malattia, in relazione alla prognosi individuale. Infine, il supporto psico-oncologico dovrebbe sempre essere offerto ai pazienti che manifestino dei bisogni non soddisfatti anche in questa patologia oncologica caratterizzata da una prognosi favorevole. 